\title{
Elaboração de diferentes tipos cookies utilizando o reaproveitamento integral dos alimentos
}

Paula Karoline Soares Farias ${ }^{1 *}$, Mateus Gomes Pereira ${ }^{2}$, Lauemy Kelly Miranda ${ }^{3}$, Ana Flávia Biondi Maia ${ }^{4}$, Katyane Benquerer Oliveira de Assis ${ }^{5}$, Adriana Benquerer Oliveira Palma ${ }^{6}$, Agda Silene Leite ${ }^{7}$, Marinilza Soares Mota Sales ${ }^{8}$, Margarete Fernandes Araújo ${ }^{9}$, Delaine Martins da Silva ${ }^{10}$

DOI: https://doi.org/10.35699/2447-6218.2020.16288

\begin{abstract}
Resumo
O objetivo foi desenvolver cookies e testar a aceitabilidade em uma comunidade acadêmica. Trata-se de um estudo quantitativo, transversal e descritivo, realizado em março de 2018, em uma faculdade privada de Montes Claros, Minas Gerais, com 200 acadêmicos não treinados. Foram desenvolvidos cookies utilizando a casca de laranja, talos de couve, casca e a semente da abóbora e a casca da banana. Foram realizados testes de aceitabilidade e intenção de compra. Verifica-se que a aceitabilidade do cookie 2 - talos de couve com $42 \%(n=84)$ com "gostei extremamente", o cookie 1 - casca de laranja com 35\% ( $n=70)$, enquanto que o cookie 3 - casca e semente de abóbora apresentou $25 \%(n=50)$ e o cookie 4 - casca de banana com $15,5 \%(n=31)$. Observa-se que os cookies desenvolvidos atendem a legislação vigente quanto ao teor de fibras, variando de 0,7 a 1,91g/20g. A intenção de compra dos cookies apresentou resultados satisfatórios, sendo que o cookie 2 - talos de couve apresentou 47,5\% (n=95) de provadores que "comprariam sempre" sendo o mais aceito pelos participantes. Os cookies elaborados demonstraram ser um produto satisfatório para o consumo, além apresentarem alto teor de fibras.
\end{abstract}

Palavras-chave: Aproveitamento Integral dos Alimentos. Alimento Funcional. Dieta Saudável.

\section{Elaboration of different types of cookies using the full reuse of food}

\footnotetext{
${ }^{1}$ Universidade Estadual de Montes Claros - Unimontes. Montes Claros, MG. Brasil. https://orcid.org/0000-0003-0529-2754

${ }^{2}$ Faculdades Integradas do Norte de Minas - FUNORTE. Montes Claros, MG. Brasil. https://orcid.org/0000-0003-3981-7838

${ }^{3}$ Faculdades Integradas do Norte de Minas - FUNORTE. Montes Claros, MG. Brasil. https://orcid.org/0000-0002-9468-6861

${ }^{4}$ Instituto Israelita de Ensino e Pesquisa Albert Einstein - IIEP. São Paulo, SP. Brasil. https://orcid.org/0000-0003-2079-8366

${ }^{5}$ Universidade Estadual de Montes Claros - Unimontes. Montes Claros, MG. Brasil. https://orcid.org/0000-0001-6178-2219

${ }^{6}$ Universidade Estadual de Montes Claros - Unimontes. Montes Claros, MG. Brasil. https://orcid.org/0000-0002-0567-3285

${ }^{7}$ Universidade Estadual de Montes Claros - Unimontes. Montes Claros, MG. Brasil. https://orcid.org/0000-0003-4825-8206

${ }^{8}$ Universidade Estadual de Montes Claros - Unimontes. Montes Claros, MG. Brasil. https://orcid.org/0000-0002-4987-3879

${ }^{9}$ Faculdades Integradas do Norte de Minas - FUNORTE. Montes Claros, MG. Brasil. https://orcid.org/0000-0001-8626-4581

${ }^{10}$ Faculdades Integradas do Norte de Minas - FUNORTE. Montes Claros, MG. Brasil. https://orcid.org/0000-0001-8030-5370

*Autor para correspondência: paulak.soares@hotmail.com
}

Recebido para publicação em 26 de dezembro de 2019. Aceito para publicação em 15 de agosto de 2020. e-ISSN: 2447-6218 / ISSN: 2447-6218. Atribuição CC BY. 
Farias, P. K. S. et al.

\begin{abstract}
The aim of the study was to develop cookies and test acceptability in an academic community. This is a quantitative, cross-sectional and descriptive study, conducted in March 2018, at a private college in Montes Claros, Minas Gerais, with 200 untrained academics. Cookies were developed using orange peel, cabbage stalks, pumpkin peel and seed and banana peel. Acceptability and purchase intention tests were performed. It turns out that the acceptability of cookie 2 - cabbage stalks with $42 \%(n=84)$ with "I liked it extremely", cookie 1 - orange peel with $35 \%(n=70)$, while cookie 3 - pumpkin peel and seed presented $25 \%(n=50)$ and cookie 4 - banana peel with $15.5 \%(n=31)$. It is observed that the cookies developed comply with the current legislation regarding fiber content, ranging from $0.7 \mathrm{to} 1.91 \mathrm{~g} / 20 \mathrm{~g}$. The intention to purchase cookies showed satisfactory results, and cookie 2 - cabbage stalks showed $47.5 \%(n=95)$ of tasters who would "always buy" being the most accepted by the participants. The cookies elaborated proved to be a satisfactory product for consumption, besides presenting high fiber content.
\end{abstract}

Keywords: Whole Utilization of Foods. Functional Food. Healthy Diet.

\section{Introdução}

O Brasil encontra-se entre os maiores produtores de alimentos e o segundo maior exportador de produtos agroalimentares em todo o mundo, mas em contrapartida o país vive a realidade entre a desnutrição e o desperdício de comida (FAO, 2018), e esses fatores representam perdas significativas para a economia do país. Toneladas de alimentos são jogadas fora diariamente, mas poderiam ser aproveitados integralmente, evitando a fome e contribuindo para a segurança alimentar (Laurindo; Ribeiro, 2014).

As estimativas mostram que aproximadamente $40 \%$ dos alimentos produzidos no mundo são desperdiçados ou perdidos em alguma fase da produção, transporte ou comercialização, com o setor do varejo sendo responsável por aproximadamente $5 \%$ das perdas nos países desenvolvidos (Sun et al., 2018). O custo econômico total do desperdício de alimentos é de US\$ 750 bilhões (a preços de produtores em 2018) ou US\$ 936 bilhões (no mercado de 2018) e é contribuído principalmente por vegetais (23\% do custo total), seguido por carne (21\%), frutas (19\%) e cereais (18\%) (Lemaire; Limbourg, 2019).

No mundo são desperdiçados mais de 13 milhões de toneladas de alimentos por ano, e na América Latina este índice chega a aproximadamente 127 mil toneladas por ano. Cada pessoa desperdiça em média $223 \mathrm{~kg}$ de alimentos na América Latina, sendo que 36 milhões de pessoas poderiam suprir as necessidades calóricas utilizando os alimentos que são desperdiçados (FAO, 2017).

Nesta perspectiva, aproveitar os alimentos que são descartados, visando à produção de um produto que apresente um menor custo, para o consumidor e forneça vitaminas e minerais adequados, torna-se atrativo. Utilizar os alimentos de maneira integral é uma forma de cooperar para a redução eficiente do resíduo orgânico, que atualmente reproduz $65 \%$ de toda sujidade gerada no país, ou seja, o aproveitamento para o uso integral dos alimentos diminui o desperdício e melhora a qualidade com pouco ou quase nenhum recurso (Farias et al., 2016).
Ao incluir em preparações partes comumente inutilizadas, abrem-se possibilidades para criações de novas receitas e incentivo ao consumo in natura das mesmas. Tais atitudes são observadas como uma forma de contribuição significativa para o enriquecimento nutricional, uma vez que muitos alimentos possuem nas partes vistas como "menos nobres", um teor elevado de nutrientes (Laurindo; Ribeiro, 2014). O reaproveitamento integral dos alimentos com a elaboração de biscoitos tipo cookie, torna-se uma alternativa viável para a utilização das cascas, sementes e talos, pois estes seriam desprezados, e estratégias como essa, torna-se uma alternativa saudável, com baixo custo, e evitam o desperdício desses alimentos (Dou et al., 2016; Storck et al., 2013; Cardoso et al., 2015; Melo; Clerici, 2013).

Sendo assim, observa-se um número crescente de pesquisas que almejam elaborar novos produtos a partir do processo de reaproveitamento integral dos alimentos com o objetivo de estimular a alimentação saudável e consciente na sociedade, podendo destacar o desenvolvimento de cookies com o aproveitamento de casca de frutas e vegetais (Dou et al., 2016; Teixeira; Oselame, 2013; Graça et al., 2016). Diante do exposto, o objetivo do estudo foi desenvolver os cookies e testar sua aceitabilidade em uma comunidade acadêmica.

\section{Material e Métodos}

O estudo caracteriza-se como transversal e quantitativo. Os cookies funcionais foram elaborados e desenvolvidos no Laboratório de Técnica e Dietética de uma faculdade privada de Montes Claros - MG. Para o desenvolvimento dos mesmos foram utilizados ingredientes adquiridos no comércio local da cidade, e utilizou-se toda a estrutura física do laboratório. O estudo foi realizado em março de 2018.

Os critérios de inclusão para a realização do teste de aceitabilidade foram: estar regularmente matriculado e frequente na faculdade escolhida para a realização da pesquisa; estar presente no dia da realização do teste de 
Elaboração de diferentes tipos cookies utilizando o reaproveitamento integral dos alimentos

aceitabilidade e aceitar participar da pesquisa de forma voluntária, com consentimento por meio da assinatura do Termo de Consentimento Livre e Esclarecido - TCLE. Os critérios de exclusão foram: não comprovar a participação na pesquisa por meio da apresentação do TCLE; não estar presente no dia da realização do teste de aceitabilidade e ser alérgico e/ou intolerante a algum dos ingredientes a serem utilizados nas receitas e/ou presentes no produto comercial que constam no TCLE apresentado aos participantes.
No laboratório foram desenvolvidos quatro sabores de cookies: cookie 1- Sabor casca de laranja; cookie 2 - Sabor talo de couve; cookie 3 - Sabor casca e semente de abóbora e o cookie 4 - Sabor casca de banana (Figura 1). Os produtos in natura (laranja, couve, abóbora e banana) foram adquiridos no comércio local e passaram por uma higienização por meio da solução de hipoclorito de sódio durante 15 minutos, sendo que para cada preparação foi atribuída uma ficha técnica com o intuito de padronizar as receitas e os cálculos.

Figura 1 - Cookies elaborados nos sabores 1 - casca de laranja (a); 2 - talos de couve (b); 3 - casca e semente de abóbora (c); 4 - casca de banana (d).

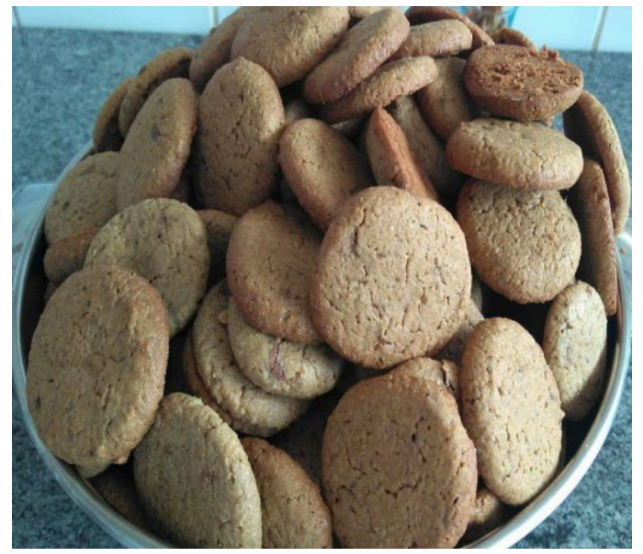

Cookie 1 - casca de laranja (a)

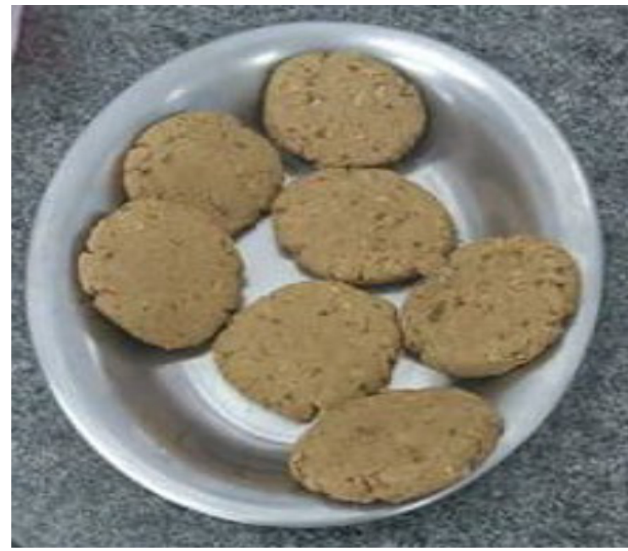

Cookie 3 - casca e semente de abóbora (c)

Posterior à higienização, foram retiradas as cascas da laranja, que foram secas em forno a $180^{\circ} \mathrm{C}$ por 2 horas e liquidificadas em forma de farinha. Os talos da couve foram picados e liquidificados até formar uma pasta. A casca e a semente de abóbora foram secas em forno a $180^{\circ} \mathrm{C}$ por 20 minutos, e em seguida trituradas e liquidificadas. A casca da banana foi cozida e liquidificada, formando uma pasta e reservada para a etapa de mistura. Após este procedimento, os alimentos foram colocados em tigelas distintas e acrescidos com os demais ingredientes constituintes das receitas, conforme descrito na Tabela 1. Os cookies foram modelados em formato de biscoito com tamanho e peso de $20 \mathrm{~g}$ cada unidade, e em seguida, foram assados em forno doméstico a $180^{\circ} \mathrm{C}$ por aproximadamente 20 minutos.

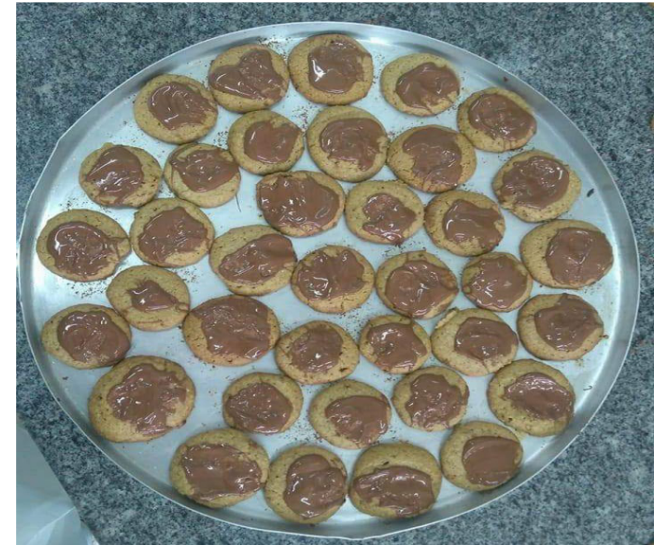

Cookie sabor 2 - talos de couve (b)

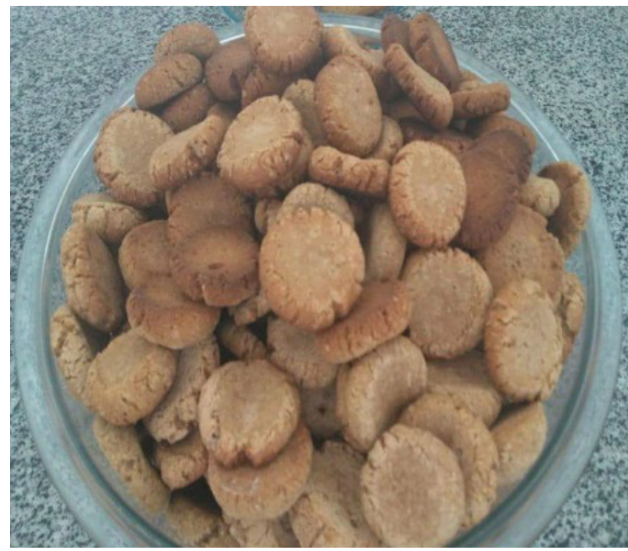

Cookie 4 - casca de banana (d)

Para a determinação do valor nutricional dos cookies desenvolvidos utilizou-se a Tabela de Composição de Alimentos (TACO, 2011), e calculados o valor energético total, os macronutrientes (Carboidratos, Proteínas e Lipídeos) e o teor de fibras.

A amostra do teste de aceitabilidade e intenção de compra foi constituída por 200 acadêmicos, não treinados, resultantes do cálculo amostral no qual o erro amostral foi de 5\% e o nível de confiança igual a 95\%. Os participantes foram de ambos os sexos, com faixa etária ente 21 a 60 anos. A seleção foi realizada de maneira aleatória, e obedeceram aos critérios de inclusão e exclusão do presente estudo. 
Farias, P. K. S. et al.

Tabela 1 - Ingredientes utilizados na elaboração dos cookies nos sabores 1 - casca de laranja; 2 - talos de couve; 3 casca e semente de abóbora; 4 - casca de banana.

\begin{tabular}{|c|c|c|c|c|}
\hline & Cookie Sabor 1 & Cookie Sabor 2 & Cookie Sabor 3 & Cookie Sabor 4 \\
\hline \multirow{7}{*}{ Ingredientes } & Farinha de arroz & Farinha de arroz & Farinha de arroz & Farinha de arroz \\
\hline & Açúcar mascavo & Açúcar mascavo & Açúcar mascavo & Açúcar mascavo \\
\hline & Aveia & Aveia & Aveia & Aveia \\
\hline & Casca de laranja seca & $\begin{array}{l}\text { Talos da couve } \\
\text { Achocolatado em pó }\end{array}$ & $\begin{array}{l}\text { Casca e semente de } \\
\text { abóbora }\end{array}$ & $\begin{array}{l}\text { Cascas das bananas } \\
\text { cozidas }\end{array}$ \\
\hline & Fermento em pó & Fermento em pó & Fermento em pó & Fermento em pó \\
\hline & Manteiga & Manteiga & Manteiga & Manteiga \\
\hline & Ovo & Ovo & Ovo & Canela \\
\hline
\end{tabular}

Os participantes receberam as quatro preparações dos cookies, que foram entregues separadamente e devidamente identificados de maneira numérica. Após a degustação dos diferentes tipos de cookies foi realizado os testes de aceitabilidade e intenção de compra. Em seguida realizou-se a explicação do preenchimento das fichas, e estas foram distribuídas e respondidas em um espaço reservado para o teste. Os participantes foram orientados a escolher o número da escala que fosse condizente com a aceitação. $O$ preenchimento das escalas foi realizado em local reservado, para evitar conversas entre os participantes e após o término as fichas preenchidas foram recolhidas.

Os dados coletados por meio do preenchimento das fichas representavam a preferência, aceitação (cor, sabor, textura), que corresponde a escala hedônica de 9 pontos na qual foi informada desde "desgostei muitíssimo" e "gostei muitíssimo" e a intenção de compra (escala de 7 pontos) variando de "compraria sempre" a "nunca compraria", sendo este formulário adaptado do Instituto Adolfo Lutz (IAL, 2008).

As respostas dos participantes foram analisadas por meio de análise estatística, e os dados foram armazenados e analisados em planilhas. Os resultados obtidos com as coletas das informações foram tabulados no programa Excel 2010 e tratados através da estatística descritiva, no qual foram apresentados em tabelas, contendo frequências e percentuais, e exportados para a análise estatística no software Statistical Package for the Social Sciences (SPSS) - 19.

O estudo seguiu as diretrizes do Conselho Nacional de Saúde através da Resolução 466/12, e o trabalho foi aprovado pelo Comitê de Ética em Pesquisa da Associação Educativa do Brasil - SOEBRAS sob o № do parecer 2.207.913 e CAAE: 69776817.8.0000.5141.

\section{Resultados e discussão}

Os biscoitos tipo cookie apresentam boa aceitação e está presente em refeições rápidas da população.
O cookie tem um valor nutricional considerável, uma vez que podem ser adicionados ingredientes benéficos ao organismo humano na formulação (Mariani et al., 2015). Verifica-se um aumento na produção de biscoitos no Brasil, com 1,7 milhão de toneladas/ano, com média de R $\$ 21$ bilhões e consumo per capita de $8,5 \mathrm{~kg} /$ ano. Sendo assim, observa-se o potencial para acrescentar novos produtos, com possibilidade de comercialização (ABIMAPI, 2016), em especial, na perspectiva de aproveitamento integral dos alimentos, visto a quantidade desperdiçada no Brasil (Laurindo; Ribeiro, 2014).

Quanto à composição química dos cookies elaborados, e segundo a Portaria 27/98 (BRASIL, 1998) a recomendação de fibras mediante a rotulagem nutricional é de no mínimo $3 \mathrm{~g} / 100 \mathrm{~g}$, sendo assim, pode-se verificar que os cookies desenvolvidos atendem a legislação vigente (tabela 2). O consumo regular de fibras é importante, pois tais compostos contribuem para a diminuição do colesterol plasmático, prevenindo doenças cardiovasculares, atuam também no combate à obesidade promovendo saciedade, e consequentemente, menor ingestão calórica, propiciam o retardo na absorção de glicose e ainda protegem contra o câncer de intestino (Vidal; Dias; Martins, 2012).

A quantidade de carboidrato nos cookies variou de 9,87 a 11,66(g). No estudo de Silva et al. (2019), ao avaliarem o teor de carboidratos totais no biscoito tipo cookie feito a partir da farinha do caroço de abacate, os autores encontraram $57,28 \%$ de carboidrato, enquanto que Nascimento et al. (2016) foi de 63,80\%. Os teores de proteínas variaram de 0,44 a 0,92(g), Silva et al. (2019), trabalharam com teores de 5,17\%, Nascimento et al. (2016) e Guimarães e Capobiango (2017) encontraram valores de $4,57 \%$ e $3,34 \%$, respectivamente. $E$ o teor de lipídeo variou de 0,88 a 1,53(g). Guimarães e Capobiango (2017) encontraram 3,03\%, e Silva et al. (2019) foi de $8,73 \%$ (tabela 2).

Desta forma, a elaboração de cookies enriquecidos nutricionalmente, com propriedades tecnológicas e sensoriais adequadas, permitiu o reaproveitamento de alimentos, além de ser de extrema importância para a 
comunidade científica, comércio e consumo geral. Verifica-se que variações nutricionais podem ocorrer, mediante a adição de diferentes alimentos e quantidades (Paz et al., 2015). A aceitabilidade do cookie 2 - sabor talos de couve conforme demonstrado na tabela 3 apresentou
$42 \%(n=84)$ de "gostei extremamente", o cookie 2 com $35 \%(n=70)$ de "gostei extremamente", enquanto que o cookie 3 apresentou 25\% $(n=50)$, e o cookie 1 com $15,5 \%(n=31)$.

Tabela 2 - Composição química dos cookies nos sabores 1 - casca de laranja, 2 - talos de couve, 3 - casca e semente de abóbora, 4 - casca de banana (1 unidade de $20 \mathrm{~g}$ ).

\begin{tabular}{cccccc}
\hline Cookie & Kcal & Carboidrato $(\mathrm{g})$ & Proteína $(\mathrm{g})$ & Lipídeo $(\mathrm{g})$ & Fibra $(\mathrm{g})$ \\
\hline Sabor 1 & 57,87 & 11,51 & 0,91 & 0,91 & 0,91 \\
Sabor 2 & 55,45 & 9,87 & 0,55 & 1,53 & 1,62 \\
Sabor 3 & 58,24 & 11,66 & 0,92 & 0,88 & 1,81 \\
Sabor 4 & 51,13 & 10,25 & 0,44 & 0,93 & 0,7 \\
\hline
\end{tabular}

No estudo de Padilha e Basso (2015) ao avaliaram os biscoitos preparados com $15 \%$ de resíduos de frutas, em substituição à farinha de trigo os resultados da análise sensorial demonstraram uma boa aceitação dos cookies, demonstrando ter uma boa aceitabilidade dentre os participantes da pesquisa, conforme encontrado neste trabalho com o cookie sabor 2. Silva et al.
(2019) ao realizaram a análise sensorial, verificaram que a formulação com adição de $5 \%$ da farinha do caroço de abacate apresentou melhor aceitação entre os provadores, procedendo em $58 \%$ de aprovação, a formulação com adição de $10 \%$ da farinha do caroço de abacate alcançou $30 \%$ da preferência dos participantes.

Tabela 3 - 'Teste de aceitabilidade dos cookies nos sabores 1 - casca de laranja, 2 - talos de couve, 3 - casca e semente de abóbora, 4 - casca de banana realizado com os provadores não treinados de uma faculdade privada de Montes Claros - MG.

\begin{tabular}{ccccccccc}
\hline \multirow{2}{*}{ Teste de Aceitabilidade } & \multicolumn{2}{c}{ Cookie Sabor $\mathbf{1}$} & \multicolumn{2}{c}{ Cookie Sabor 2 } & \multicolumn{2}{c}{ Cookie Sabor 3 } & \multicolumn{2}{c}{ Cookie Sabor 4 } \\
& $\mathbf{n}$ & $\mathbf{\%}$ & $\mathbf{n}$ & $\mathbf{\%}$ & $\mathbf{n}$ & $\mathbf{\%}$ & $\mathbf{n}$ & $\mathbf{\%}$ \\
\hline Desgostei extremamente & 1 & 0,5 & 2 & 1 & 6 & 3 & 18 & 9 \\
Desgostei moderadamente & 4 & 2 & 4 & 2 & 2 & 1 & 8 & 4 \\
Desgostei regularmente & 0 & 0 & 8 & 4 & 5 & 2,5 & 9 & 4,5 \\
Desgostei ligeiramente & 5 & 2,5 & 2 & 1 & 10 & 5 & 7 & 3,5 \\
Não gostei, nem desgostei & 9 & 4,5 & 14 & 7 & 12 & 6 & 19 & 9,5 \\
Gostei ligeiramente & 20 & 10 & 17 & 8,5 & 34 & 17 & 36 & 18 \\
Gostei regularmente & 35 & 17,5 & 29 & 14,5 & 36 & 18 & 34 & 17 \\
Gostei moderadamente & 56 & 21 & 40 & 20 & 45 & 22,5 & 38 & 19 \\
Gostei extremamente & 70 & 35 & 84 & 42 & 50 & 25 & 31 & 15,5 \\
\hline
\end{tabular}

A intenção de compra dos cookies apresentou resultados satisfatórios com o cookie no sabor 2 apresentando 47,5\% (n=95) de provadores que "comprariam sempre", sendo o mais aceito pelos participantes, o cookie sabor 1 apresentou 35\% $(n=70)$, o cookie sabor 3 com $25 \%(n=25 \%)$, e o sabor 4 representou $22 \%(n=44)$ (tabela 4). Silva et al. (2019) avaliaram que a formulação com adição de $20 \%$ da farinha do caroço de abacate ao comparar com a farinha de trigo, esta foi a menos aceita entre os provadores (14\%).

Dessa forma, pode-se indicar os cookies elaborados nos sabores de casca de banana; casca de laranja; casca e semente de abóbora; e talos de couve, como alternativas importantes por serem nutritivas e uma opção proveitosa para as indústrias que descartam esses alimentos, em especial, por considerarem que estes alimentos apresentam baixo valor financeiro. Além disso, destaca-se a importância para a produção de alimentos destinados a aproveitar o alimento de maneira integral, pois apresentam valores calóricos e de macronutrientes baixos, e são ricos em vitaminas e minerais, além de demonstrarem bons níveis de aceitação e intenção de compra (tabela 3 e 4). 
Tabela 4 - Teste de Intenção de compra dos cookies nos sabores 1 - casca de laranja, 2 - talos de couve, 3 - casca e semente de abóbora, 4 - casca de banana realizado com os provadores não treinados de uma faculdade privada de Montes Claros - MG.

\begin{tabular}{ccccccccc}
\hline \multirow{2}{*}{$\begin{array}{c}\text { Teste de Intenção de } \\
\text { Compra }\end{array}$} & \multicolumn{2}{c}{ Cookie $\mathbf{1}$} & \multicolumn{2}{c}{ Cookie $\mathbf{2}$} & \multicolumn{2}{c}{ Cookie 3 } & \multicolumn{2}{c}{ Cookie 4 } \\
\hline Nunca compraria & 2 & 1 & 1 & 0,5 & 9 & 4,5 & 18 & 9 \\
Compraria muito raramente & 9 & 4,5 & 16 & 8 & 9 & 4,5 & 29 & 14,5 \\
$\quad$ Compraria raramente & 11 & 5,5 & 14 & 7 & 19 & 9,5 & 23 & 11,5 \\
Compraria ocasionalmente & 30 & 15 & 33 & 16,5 & 41 & 20,5 & 45 & 22,5 \\
Compraria frequentemente & 45 & 22,5 & 19 & 9,5 & 33 & 16,5 & 19 & 9,5 \\
$\quad \begin{array}{c}\text { Compraria muito } \\
\text { frequentemente }\end{array}$ & 36 & 18 & 22 & 11 & 25 & 12,5 & 22 & 11 \\
$\quad \begin{array}{c}\text { Compraria sempre } \\
\text { n }\end{array}$ & 67 & 33,5 & 95 & 47,5 & 64 & 32 & 44 & 22 \\
\hline
\end{tabular}

Verificou-se com a realização do teste de intenção de compra a possibilidade de comercialização dos cookies nos sabores de casca de banana; casca de laranja; casca e semente de abóbora; e talos de couve, torna-os viável e uma alternativa saudável, além de contribuir com o aproveitamento integral dos alimentos, sendo uma opção para a utilização do alimento de forma integral, contribuindo assim para evitar o desperdício, e observou-se uma boa aceitação em todas as formulações desenvolvidas.

\section{Conclusão}

Pode-se observar que a elaboração e preparo dos diferentes tipos de cookies utilizando o reaproveitamento integral dos alimentos apresentou uma boa aceitação entre os participantes, demostrando ser um produto satisfatório para o consumo. Os cookies apresentaram alto teor de fibras, sendo uma ótima alternativa para aumentar a ingestão na alimentação. Verifica-se que este trabalho demonstra alternativas para a utilização do alimento de maneira integral, em especial para os testados nesta pesquisa. Como limitações, apresenta a ausência de realização das análises físico-químicas para a quantificação real dos cookies elaborados.

\section{Referências}

Associação Brasileira das Indústrias de Biscoitos, Massas Alimentícias e Pães \& Bolos Industrializados (ABIMAPI). Categorias de biscoitos, massas alimentícias, pães e bolos industrializados fecham 2015 com faturamento de R \$ 35,4 bilhões [Internet]. 2016. Disponível em: https:// www.abimapi.com.br/noticias-detalhe.php? $\mathrm{i}=\mathrm{MTc} 1 \mathrm{OQ}==$ Acesso $\mathrm{em}$ 26 nov de 2019.

Brasil. Portaria no 27, de 13 de janeiro de 1998. Disponível em: http:// portal.anvisa.gov.br/documents/33916/394219/PORTARIA 271998. pdf/72db7422-ee47-4527-9071-859f1f7a5f29 Acesso em 11 ago de 2019.

Cardoso, F. T.; Fróes, S. C.; Friede, R.; Moragas, C. J.; Miranda, M. G.; Avelar, K. E. S. 2015. Aproveitamento integral de Alimentos e o seu impacto na Saúde. Sustentabilidade em Debate, 6(3): 131-143. Doi: https://doi.org/10.18472/SustDeb.v6n3.2015.16105.

Dou, Z.; Ferguson, J. D.; Galligan, D. T.; Kelly, A. M. Finn, S. M.; Giegengack, R. 2016. Assessing U.S. food wastage and opportunities for reduction. Global Food Security, 8: 19-26. Doi: https://doi. org/10.1016/j.gfs.2016.02.001.

Food and Agriculture Organization of the United Nations. FAO. The state of food security and nutrition in the world. 2018. Disponível em http://www.fao.org/3/i9553en/i9553en.pdf Acesso em 16 out de 2019.
Food and Agriculture Organization of the United Nations. FAO. The Future and Food and Agriculture: Trends and Challenges. 2017. Disponível em: http://www.fao.org/3/a-i6583e.pdf Acesso em 16 out de 2019.

Farias, P. K. S.; Souza, S. D. O.; Santana, I. M. O.; Prates, R. P.; Gusmão, A. C. M.; Soares, P. D. F.; 2016. Desenvolvimento e análise sensorial de diferentes tipos de hambúrgueres funcionais utilizando o reaproveitamento de alimentos. Caderno de Ciências Agrárias, 8(3): 07-14.

Graça, P.; Souza, S. M.; Correia, A.; Salvador, C.; Filipe, J.; Carriço, J.; Gregóri, M. J. 2016. Alimentação Saudável em Números. DireçãoGeral da Saúde, p. 1-100. Disponível em: http://hdl.handle. net/10400.26/15566 Acesso em 03 nov de 2019.

Guimarães, P. B.; Capobiango, M. Determinação da composição centesimal da farinha obtida a partir da farinha do caroço de abacate. In Anais do III Congresso Nacional de Alimentos e Nutrição e VI Congresso Nacional de Alimentação e Nutrição. 2017. Ouro Preto: PUC Minas.

Instituto Adolfo Lutz. Análise Sensorial. São Paulo, 2008. Versão eletrônica. Disponível em http://www.ial.sp.gov.br/index. php?option $=$ com_remository\&ltemid $=20 \&$ finc $=$ fileinfo\&id $=7$ Acesso em 17 ago de 2019. 
Laurindo, T. R.; Ribeiro, K. R. R. 2014. Aproveitamento Integral de Alimentos. Interciência e Sociedade, 3(2): 17-26.

Lemaire, A.; Limbourg, S. 2019. How can food loss and waste management achieve sustainable development goals? Journal of Cleaner Production, 234: 1221-1234. Doi: https://doi.org/10.1016/j. jclepro.2019.06.226.

Mariani, M.; Oliveira, V. R.; Faccin, R.; Rios, A. O.; Venzke, J. G. 2015. Elaboração e avaliação de biscoitos sem glúten a partir de farelo de arroz e farinhas de arroz e de soja. Brazilian Journal of Food Technology, 1: 70-78. Doi: http://dx.doi.org/10.1590/1981-6723.6514.

Melo, L. S. M.; Clerici, M. T. P. 2013. Desenvolvimento e avaliação tecnológica, sensorial e físico-química de produto cárneo, tipo hambúrguer, com substituição de gordura por farinha desengordurada de gergelim. Brazilian Journal of Food and Nutrition. 24(4): 361-368.

Nascimento, M. R. F.; Souza, V. F.; Marinho, A. F.; Ascheri, J. L. R.; Meleiro, C. H. 2016. Composição centesimal e minerais de farinha do caroço de abacate (Persea gratissima, Gaertner f.). In Anais do XXV Congresso Brasileiro de Ciência e Tecnologia de Alimentos; X CIGR Session VI International Technical Symposium. Gramado: SBCTA Regional. Disponível em https://www.alice.cnptia.embrapa.br/ bitstream/doc/1057908/1/67.pdf. Acesso em 24 nov de 2019.

Padilha, T.; Basso, C. 2015. Biscoitos com resíduos de manga, maracujá e jabuticaba. Disciplinarum Scientia. Série Ciências Biológicas e da Saúde, 16(1): 79-88.
Paz, M. F.; Marques, R. V.; Schumann, C.; Corrêa, L. B.; Corrêa, E. K. 2015. Características tecnológicas de pães elaborados com farelo de arroz desengordurado. Brazilian Journal of Food Technology, 18(2): 128-136. Doi: http://dx.doi.org/10.1590/1981-6723.6014.

Silva, I. G.; Andrade, A. P. C.; Silva, L. M. R.; Gomes, D. S. 2019. Elaboração e análise sensorial de biscoito tipo cookie feito a partir da farinha do caroço de abacate. Brazilian Journal of Food Technology, 22: 1-10. Doi: http://dx.doi.org/10.1590/1981-6723.20918.

Storck, C. R.; Nunes, G. L., Oliveira, B. B.; Basso, C. 2013. Folhas, talos, cascas e sementes de vegetais: composição nutricional, aproveitamento na alimentação e análise sensorial de preparações. Ciência Rural, 43(3): 537-543. Doi: http://dx.doi.org/10.1590/S0103-84782013000300027.

Sun, S. K.; Lu, Y. J.; Gao, H.; Jiang, T. T.; Du, X. Y.; Shen, T. X.; Wu, P. T.; Wang, Y. B. 2018. Impacts of food wastage on water resources and environment in China. Journal of Cleaner Production, 185: 732-739. Doi: https://doi.org/10.1016/j.jclepro.2018.03.029.

Tabela de Composição dos Alimentos. TACO. 2011. Campinas: NEPAUNICAMP, p. 1-161. Disponível em http://www.cfn.org.br/wp-content/ uploads/2017/03/taco_4_edicao_ampliada_e_revisada.pdf Acesso em 11 jul de 2019.

Teixeira, A. C. M.; Oselame, C. S. 2013. O uso de alimentos funcionais no cotidiano e seus benefícios a saúde. Revista Científica do Colégio Militar de Curitiba, 5(1): 65-76.

Vidal, A. M.; Dias, D. O.; Martins, E. S. M. 2012. A ingestão de alimentos funcionais e sua contribuição para diminuição da incidência de doenças. Cadernos de Graduação - Ciências Biológicas e da Saúde, 1(15): 43-52. 